\title{
On cubic planar hypohamiltonian and hypotraceable graphs
}

\author{
Makoto Araya \\ Department of Computer Science \\ Shizuoka University, Hamamatsu 432-8011, Japan \\ araya@inf.shizuoka.ac.jp \\ Gábor Wiener \\ Department of Computer Science and Information Theory \\ Budapest University of Technology and Economics \\ 1117 Budapest, Magyar tudósok körútja 2. \\ wiener@cs. bme.hu \\ Submitted: Aug 4, 2009; Accepted: Apr 5, 2011; Published: Apr 14, 2011 \\ Mathematics Subject Classification: 05C10, 05C38, 05C45

\begin{abstract}
We present a cubic planar hypohamiltonian graph on 70 vertices, improving the best known bound of 94 by Thomassen and derive some consequences concerning longest paths and cycles of planar 3-connected graphs. We also show that cubic planar hypohamiltonian graphs on $n$ vertices exist for every even number $n \geq 86$ and that cubic planar hypotraceable graphs exist on $n$ vertices for every even number $n \geq 356$, settling an open question of Holton and Sheehan.
\end{abstract}

\section{Introduction}

Hamiltonian properties of planar cubic graphs have been investigated extensively since Tait's attempt to prove the four color conjecture based on the proposition that every 3-connected planar cubic graph has a Hamiltonian cycle. This proposition was disproved by Tutte [17] in 1946. However, until 1968, when Grinberg [4] proved a simple, yet very useful theorem, such graphs were quite difficult to find. 
Theorem 1.1. (Grinberg, 1968)

Suppose a plane graph has a Hamiltonian cycle, such that there are $f_{i} i$-gons in the exterior of the Hamiltonian cycle and $f_{i}^{\prime} i$-gons in the interior of the Hamiltonian cycle. Then

$$
\sum_{i}(i-2)\left(f_{i}-f_{i}^{\prime}\right)=0 .
$$

The theorem can be used to create non-hamiltonian planar cubic graphs: suppose for example that all but one faces of a plane graph have degree congruent to 2 modulo 3 , then (1) clearly cannot hold, so the graph cannot be Hamiltonian. Since 1968, several non-hamiltonian 3-connected planar cubic graphs have been found, the smallest of them is the Barnette-Bosák-Lederberg graph on 38 vertices [2], [10], see also [5]. In 1986, Holton and McKay [7] (extending the results of many researchers) showed that there exists no 3 -connected planar non-hamiltonian cubic graph on fewer vertices.

A graph $G$ is hypohamiltonian (resp. hypotraceable) if it is not Hamiltonian (resp. traceable) but every vertex-deleted subgraph of $G$ is Hamiltonian (resp. traceable). The study of hypohamiltonian graphs started with the paper of Sousselier [9] in 1963 and since then hypohamiltonian graphs were extensively studied by many authors, see for example the survey by Holton and Sheehan [8] and the papers [12], [13], [14], [15], [16], [3], [21].

Chvátal [3] raised the question in 1973 whether there exists a cubic planar hypohamiltonian graph. This was answered by Thomassen [16], who found a sequence of such graphs on $94+4 m$ vertices for every integer $m \geq 0$ in 1981 . However, the question whether there exist smaller cubic hypohamiltonian graphs and whether there exists a positive integer $N$, such that for every integer $n \geq N$ there exists a cubic planar hypohamiltonian graph on $n$ vertices remained open (see [8]). From the results of Aldred et al. [1] follows that there is no cubic planar hypohamiltonian graph on 42 or fewer vertices. Here we present a cubic planar hypohamiltonian graph on 70 vertices. Using the method of Thomassen for creating an $n+4$ vertex cubic hypohamiltonian graph from an $n$ vertex cubic hypohamiltonian graph [16] this also shows that cubic planar hypohamiltonian graphs on $70+4 m$ vertices exist for every even integer $m \geq 0$. Since $70 \equiv 94(\bmod 4)$, this is not enough to answer the second open question, however we also give a cubic planar hypohamiltonian graph on 88 vertices, which shows that cubic planar hypohamiltonian graphs on $n$ vertices exist for every even number $n \geq 86$. Using our graphs on 70 and 88 vertices and another construction method of Thomassen [12], we can also show that a cubic planar hypotraceable graph exists on 340 vertices and on $n$ vertices for every even number $n \geq 356$.

T. Zamfirescu [21] denoted the smallest number of vertices of a planar $k$-connected graph, in which every $j$ vertices are omitted by some longest cycle (path) by $\overline{C_{k}^{j}}\left(\overline{P_{k}^{j}}\right)$. The best known bounds on the numbers $\overline{C_{3}^{2}}$ and $\overline{P_{3}^{2}}$ are also improved using our 70 -vertex graph.

We will refer to an edge between vertices $a$ and $b$ as edge $(a, b)$ and a cycle through the vertices $a_{1}, a_{2}, \ldots, a_{k}$ as cycle $\left(a_{1}, a_{2}, \ldots, a_{k}\right)$. We use the shorthand notation $G-v$ for the graph obtained from $G$ by deleting the vertex $v$. 


\section{Cubic planar hypohamiltonian graphs on 70 and 88 vertices}

In this section we present our cubic planar hypohamiltonian graphs on 70 and 88 vertices. It is worth mentioning that there exists no cubic planar hypohamiltonian graph on 42 or fewer vertices, by the results of Aldred et al. [1]. They showed that every 3-connected, cyclically 4-connected cubic planar graph has at least 42 vertices and presented all such graphs on exactly 42 vertices. Since hypohamiltonian graphs are easily seen to be 3connected and cyclically 4-connected, they must have at least 42 vertices in the cubic case. Moreover, all 42-vertex graphs presented in [1] have exactly one face with a degree not congruent to 2 modulo 3 , and it is easy to see that cubic graphs with this property cannot be hypohamiltonian, as was observed by Thomassen [12]. The size of the smallest cubic planar hypohamiltonian graph is therefore at least 44 and at most 70 . The next claims (that are extensions of the observation of Thomassen) may help to obtain a better lower bound. Let us denote the number of edges of a face $T$ by $d(T)$ and for the sake of simplicity let us call a face $F$ an $i$-face $(i=0,1,2)$, if $d(F) \equiv i(\bmod 3)$ and call the 0 and 1-faces together non-2-faces.

Claim 2.1. A cubic planar hypohamiltonian graph has at least three non-2-faces.

Proof. Let $D$ be an arbitrary cubic planar hypohamiltonian graph. If $D$ has only 2-faces, then the deletion of any vertex gives a graph $D^{\prime}$ with exactly one non-2-face, so $D^{\prime}$ is not Hamiltonian, a contradiction. $D$ cannot have exactly one non-2-face by the observation of Thomassen [12]. So let us assume that $D$ has two non-2-faces $A$ and $B$. It is easy to see that both $A$ and $B$ should be 0 -faces, because the deletion of a vertex that is in one 1-face and two 2-faces gives a graph with exactly one non-2-face. Now the deletion of a vertex not in any of the 0-faces, but adjacent to a vertex that is in exactly one of the 0 -faces gives a graph with exactly three 0-faces, of which two have two common edges. These cannot be on the same side of a Hamiltonian cycle, therefore (1) cannot be satisfied, which finishes the proof.

The following claim can be proved similarly.

Claim 2.2. If a cubic planar hypohamiltonian graph has exactly three non-2-faces, then the three non-2-faces do not have a common vertex, moreover two 1-faces or a 1-face and a 0-face cannot be adjacent.

Now let us turn our attention to the cubic planar hypohamiltonian graphs $G$ and $H$ that can be seen in Figure 1 and Figure 2, respectively.

Theorem 2.3. $G$ and $H$ are cubic planar hypohamiltonian graphs.

Proof. Both $G$ and $H$ are obviously cubic and planar. Both have one face of degree 4, and four faces of degree 7 , such that the face of degree 4 is adjacent to all faces of degree 7 and the degrees of the other faces are congruent to 2 modulo 3. By Proposition 2.1. of 


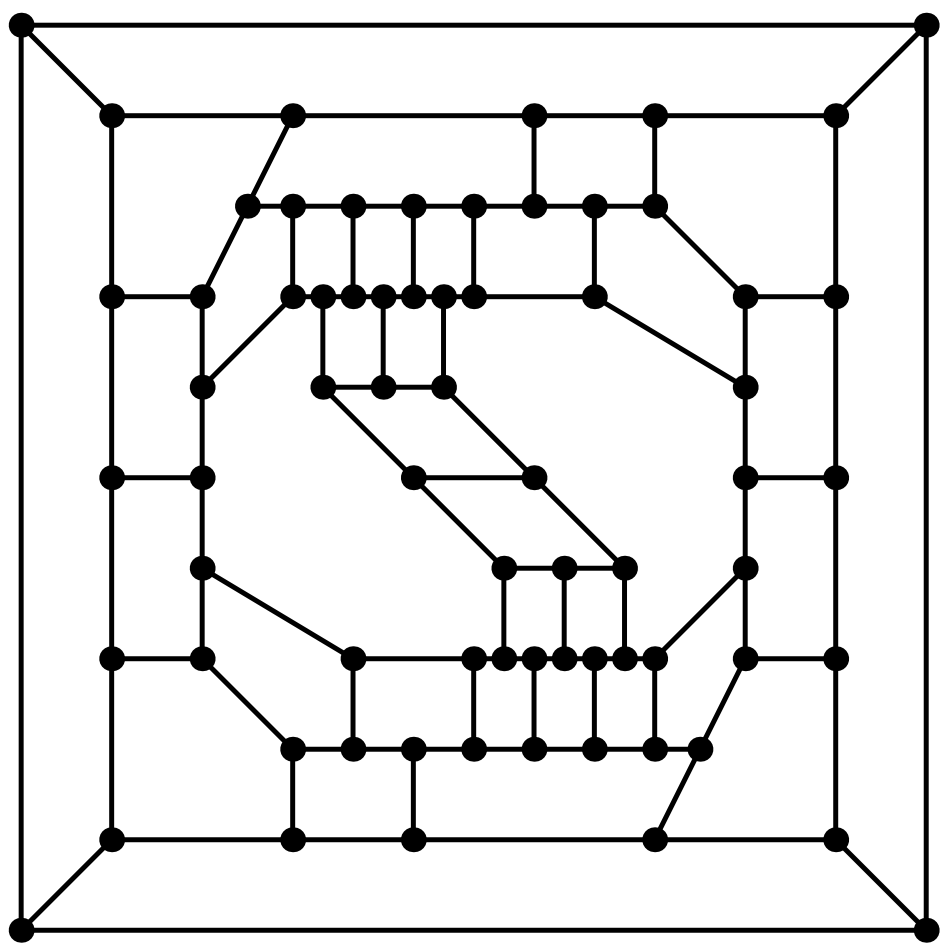

Figure 1: The cubic planar hypohamiltonian graph $G$ on 70 vertices

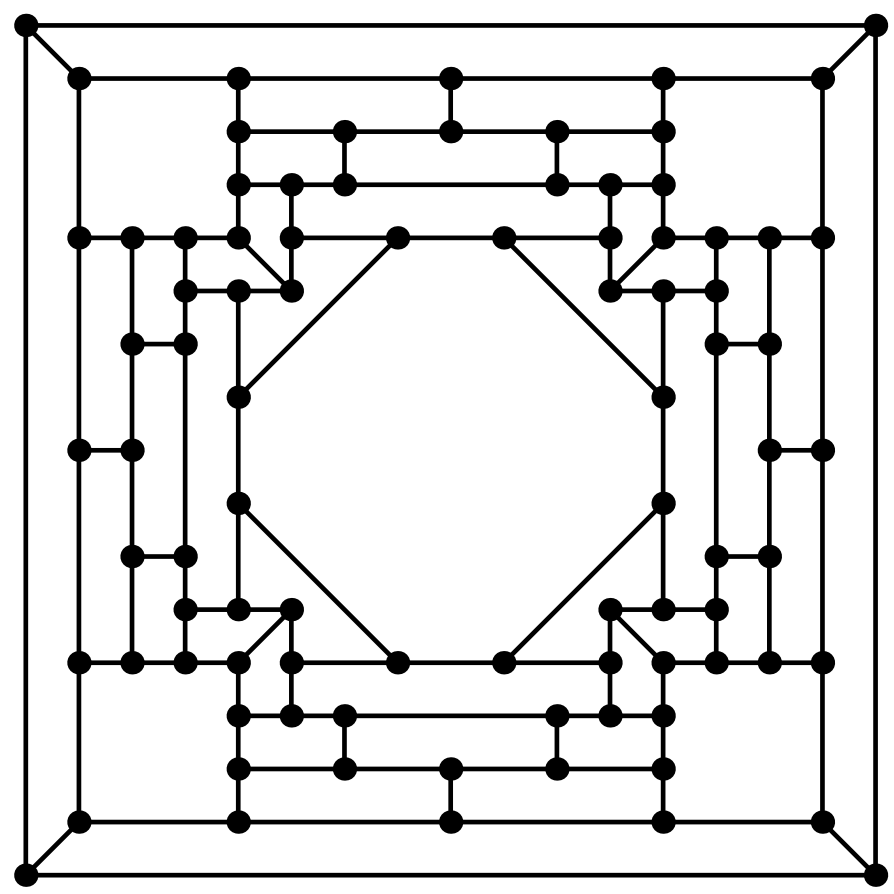

Figure 2: The cubic planar hypohamiltonian graph $H$ on 88 vertices 
[16], $G$ and $H$ are non-hamiltonian (the proof is quite easy using Grinberg's theorem: in order to satisfy (1) modulo 3, a Hamiltonian cycle should separate one of the five faces of degree 4 or 7 from the others, which is impossible in the case of $G$ and $H$ ).

Now it remains to show that every vertex-deleted subgraph of $G$ and $H$ is Hamiltonian. Since $G$ is centrally symmetric, we have to check 35 cases. $H$ has more symmetries, thus we have to check only 14 cases. The Hamiltonian cycles of the vertex-deleted subgraphs of $G$ and $H$ are presented in the last section, on Figures 3, 4, 5 and on Figure 6.

\section{Corollaries}

The most important corollary is the existence of cubic planar hypohamiltonian graphs on $n$ vertices for every even number $n \geq 86$. This settles an open question in [8].

Corollary 3.1. There exists a cubic planar hypohamiltonian graph on $n$ vertices for every even number $n \geq 86$.

Proof. The proof is quite obvious using a method of Thomassen [16]. Let $T$ be a cubic planar hypohamiltonian graph on $n$ vertices having a 4-cycle $(a, b, c, d)$. The graph $T^{\prime}$ obtained from $T$ by deleting the edges $(a, b)$ and $(c, d)$ and adding a new 4-cycle $\left(a^{\prime}, b^{\prime}, c^{\prime}, d^{\prime}\right)$ and the edges $\left(a, a^{\prime}\right),\left(b, b^{\prime}\right),\left(c, c^{\prime}\right),\left(d, d^{\prime}\right)$ to $T$. Now it is easy to see that $T^{\prime}$ is also a cubic planar hypohamiltonian graph on $n+4$ vertices having a 4-cycle. By applying this operation iteratively on the graphs $G$ and $H$ we obtain cubic planar hypohamiltonian graphs on $n$ vertices for every even number $n \geq 86$.

Using another construction of Thomassen [14] a similar corollary for hypotraceable graphs can also be proved.

Corollary 3.2. There exists a cubic planar hypotraceable graph on 340 vertices and on $n$ vertices for every even number $n \geq 356$.

Proof. We use a construction of Thomassen [14]. Let $T_{1}, T_{2}, T_{3}, T_{4}, T_{5}$ be cubic planar hypohamiltonian graphs and let $x_{i}$ and $y_{i}$ be adjacent vertices of $T_{i}(i=1,2,3,4,5)$. Let furthermore the neighbours of $x_{i}$ (resp. $y_{i}$ ), other than $y_{i}$ (resp. $x_{i}$ ) be $a_{i}$ and $b_{i}$ (resp. $c_{i}$ and $d_{i}$ ). Consider the disjoint union of the graphs $T_{i}-\left\{x_{i}, y_{i}\right\}$ and add to this graph the edges $\left(c_{1}, a_{2}\right),\left(c_{2}, a_{3}\right),\left(c_{3}, a_{4}\right),\left(c_{4}, a_{5}\right),\left(c_{5}, a_{1}\right)$ and the edges $\left(d_{1}, b_{2}\right),\left(d_{2}, b_{3}\right),\left(d_{3}, b_{4}\right)$, $\left(d_{4}, b_{5}\right),\left(d_{5}, b_{1}\right)$. Now the resulting graph $T$ is easily seen to be planar and cubic and by Lemma 3.1. of [14], it is also hypotraceable. If we choose each $T_{i}$ to be isomorphic with $G$, then we obtain a cubic planar hypotraceable graph on 340 vertices. To obtain a cubic planar hypotraceable graph on $2 k$ vertices for any $k \geq 178$ we just have to change $T_{1}$ in this construction to a cubic planar hypohamiltonian graph on $2 k-270$ vertices (such a graph exists by Corollary 3.1 , since $2 k-270 \geq 86$ ).

The next corollaries concern planar 3-connected graphs, in which every two vertices or edges are omitted by some longest cycle or path. First we improve a theorem of Schauerte and C. Zamfirescu. In [11] they showed (using a computer) that for any pair of edges $e, f$ 
there exists a longest cycle in Thomassen's 94-vertex cubic planar hypohamiltonian graph [16] avoiding $e$ and $f$. Using this observation and a method of T. Zamfirescu [21] they proved that there exists a cubic planar 3-connected graph on 8742 vertices, such that any pair of vertices is missed by a longest cycle.

The same property can also be checked easily for graph $G$ by a computer, i.e. using a software like Mathematica or Maple.

Proposition 3.3. Let $e$ and $f$ be arbitrary edges of $G$. Then there exists a longest cycle in $G$ that does not contain $e$ and $f$.

Corollary 3.4. There exists a cubic planar 3-connected graph on 4830 vertices, such that any pair of vertices is missed by a longest cycle.

Proof. We create a graph with the desired properties using a method of T. Zamfirescu [21]. Consider the 70-vertex cubic planar hypohamiltonian graph $G$, and let $V(G)=$ $\left\{a_{1}, a_{2}, \ldots, a_{70}\right\}$. Let furthermore $G^{\prime}$ be the graph obtained from $G$ by the deletion of $a_{70}$ and assume that the neighbours of $a_{70}$ are $a_{1}, a_{2}$, and $a_{3}$ in $G$. Now consider the graph $Z$ consisting of 70 copies of $G^{\prime}: G_{1}^{\prime}, G_{2}^{\prime}, \ldots, G_{70}^{\prime}$, such that we draw an edge between two copies $G_{i}^{\prime}$ and $G_{j}^{\prime}$ if and only if $a_{i}$ and $a_{j}$ are adjacent in $G$. These additional edges are always drawn between two vertices having degree 2 in the copies (that is, copies of $a_{1}, a_{2}$, or $\left.a_{3}\right)$. It is easy to see that $Z$ is a cubic planar 3 -connected graph on $69 \cdot 70=4830$ vertices. By Theorem 2.3, Proposition 3.3, and a theorem of T. Zamfirescu [21], any pair of vertices is missed by a longest cycle in $Z$. For completeness' sake we reformulate here the proof of Zamfirescu. Since $G$ is hypohamiltonian, it is easy to see that the longest cycle of $Z$ has length $68 \cdot 69=4692$ (one copy and one vertex of every other copy must be avoided, otherwise $G$ would be Hamiltonian, and a cycle of length 4692 is easy to find using the hypohamiltonicity of $G$ ). If the two vertices $x$ and $y$ we would like to avoid by a longest cycle are in the same copy, then simply consider a longest cycle avoiding this copy completely. Thus we may assume that $x$ and $y$ are in different copies. It is easy to see that there is a Hamiltonian path between two of the vertices $a_{1}, a_{2}, a_{3}$ in every vertex-deleted subgraph of $G^{\prime}$. Let $x^{\prime}\left(y^{\prime}\right)$ be that copy of $a_{1}, a_{2}$, or $a_{3}$ that is not the endvertex of such a Hamiltonian path if we delete $x(y)$. Now let us delete $x, y$, and one vertex from every other copy of $G^{\prime}$ from $Z$. Let us delete furthermore the additional edges incident to $x^{\prime}$ and $y^{\prime}$. By Theorem 2.3 and Proposition 3.3 there is a cycle of length 4692 in the remaining graph, which proves the corollary.

The following definition is due to T. Zamfirescu [20].

Definition 3.5. $\overline{C_{k}^{j}}$ (resp. $\overline{P_{k}^{j}}$ ) is the smallest number of vertices of a planar $k$-connected graph, in which every $j$ vertices are omitted by some longest cycle (resp. path).

T. Zamfirescu gave several bounds on these numbers in [21], of which the upper bounds on $\overline{C_{3}^{2}}$ and $\overline{P_{3}^{2}}$ were improved in [6] (see also [18]), [22], and [19]. The currently known best upper bounds are $\overline{C_{3}^{2}} \leq 3701, \overline{P_{3}^{2}} \leq 14694$. Using our graph $G$ we can derive even better bounds. For the proof we use the same method of T. Zamfirescu [21] as was used in [6], [22], and [19]. 
Corollary 3.6. $\overline{C_{3}^{2}} \leq 2765, \overline{P_{3}^{2}} \leq 10902$.

Proof. The method is similar to the one used in Corollary 3.4. Let $\Gamma$ be the planar hypohamiltonian graph on 42 vertices given in [19]. The graph $\Gamma^{\prime}$ is obtained by deleting any vertex of degree 3 from $\Gamma$. Now consider the graph $Y$ consisting of 70 copies of $\Gamma^{\prime}$ : $\Gamma_{1}^{\prime}, \Gamma_{2}^{\prime}, \ldots, \Gamma_{70}^{\prime}$, such that we draw an edge between two copies $\Gamma_{i}^{\prime}$ and $\Gamma_{j}^{\prime}$ if and only if $a_{i}$ and $a_{j}$ are adjacent in $G$. These additional edges are always drawn between two vertices that are copies of the neighbours of the deleted vertex. It is easy to see that $Y$ is a planar 3 -connected graph on $41 \cdot 70=2870$ vertices. From the hypohamiltonicity of $\Gamma$ and $G$, Proposition 3.3, and the mentioned theorem of Zamfirescu [21], any pair of vertices is missed by a longest cycle in $Y$. None of these properties are lost if we now contract the additional edges of $Y$ (see [21]), obtaining a graph on $41 \cdot 70-105=2765$ vertices, which proves the first upper bound.

The second bound is proved similarly. First we take four copies of $G^{\prime}$ and an additional edge between any two copies (these edges are drawn between copies of $a_{1}, a_{2}$, or $a_{3}$ again). Denote the graph obtained in this way by $X$. Now we execute the same procedure as above, but this time we put the copies of $\Gamma^{\prime}$ into the graph $X$ and then contract the additional edges to obtain a 3-connected planar graph, where every pair of vertices is missed by a longest path in $69 \cdot 4 \cdot 41-((105-3) \cdot 4+6)=10902$ vertices (see [21]).

\section{Hamiltonian cycles of the vertex-deleted subgraphs of $G$ and $H$}

In this section we present the Hamiltonian cycles of the vertex-deleted subgraphs of $G$ and H.G is centrally symmetric, so we have to check 35 cases (Figures 3, 4, and 5). $H$ has more symmetries, thus we have to check 14 cases (Figure 6).
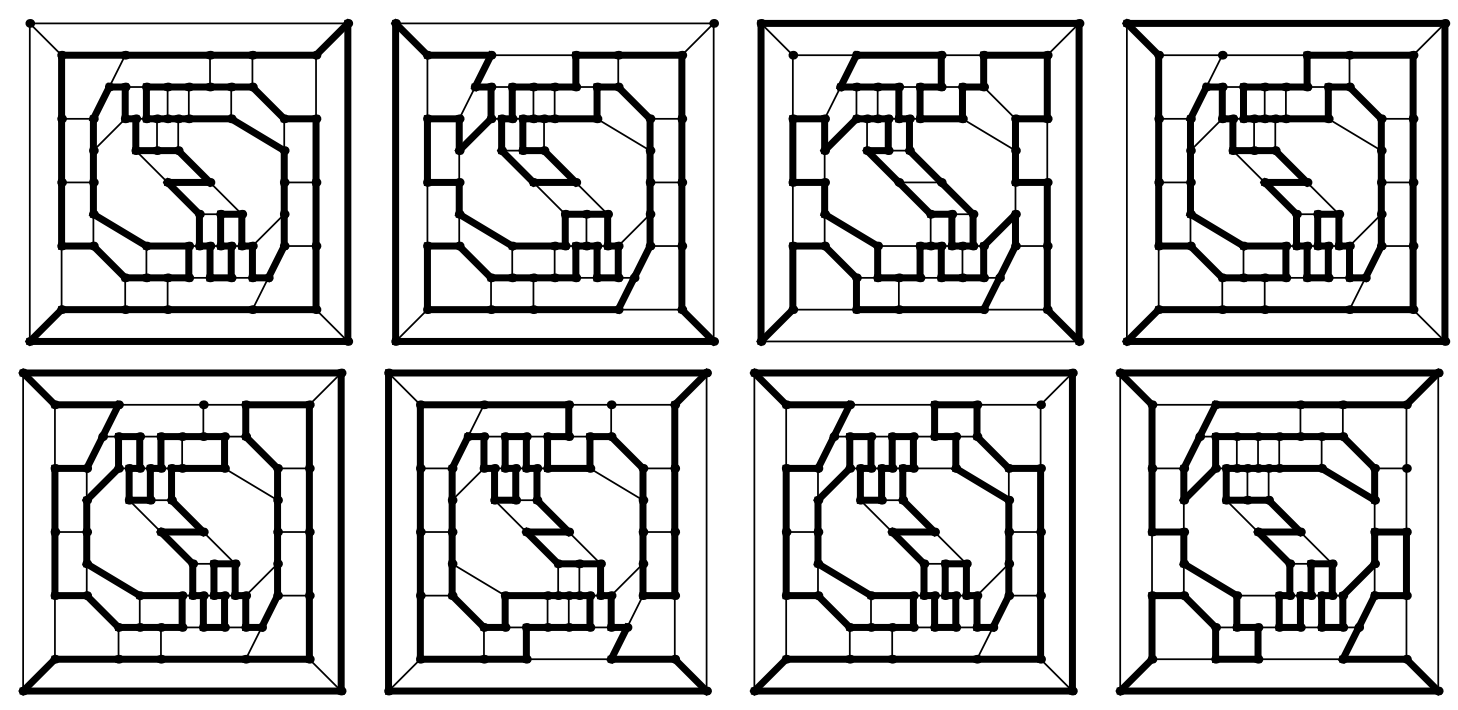

Figure 3: Hamiltonian cycles of the vertex-deleted subgraphs of $G$ 

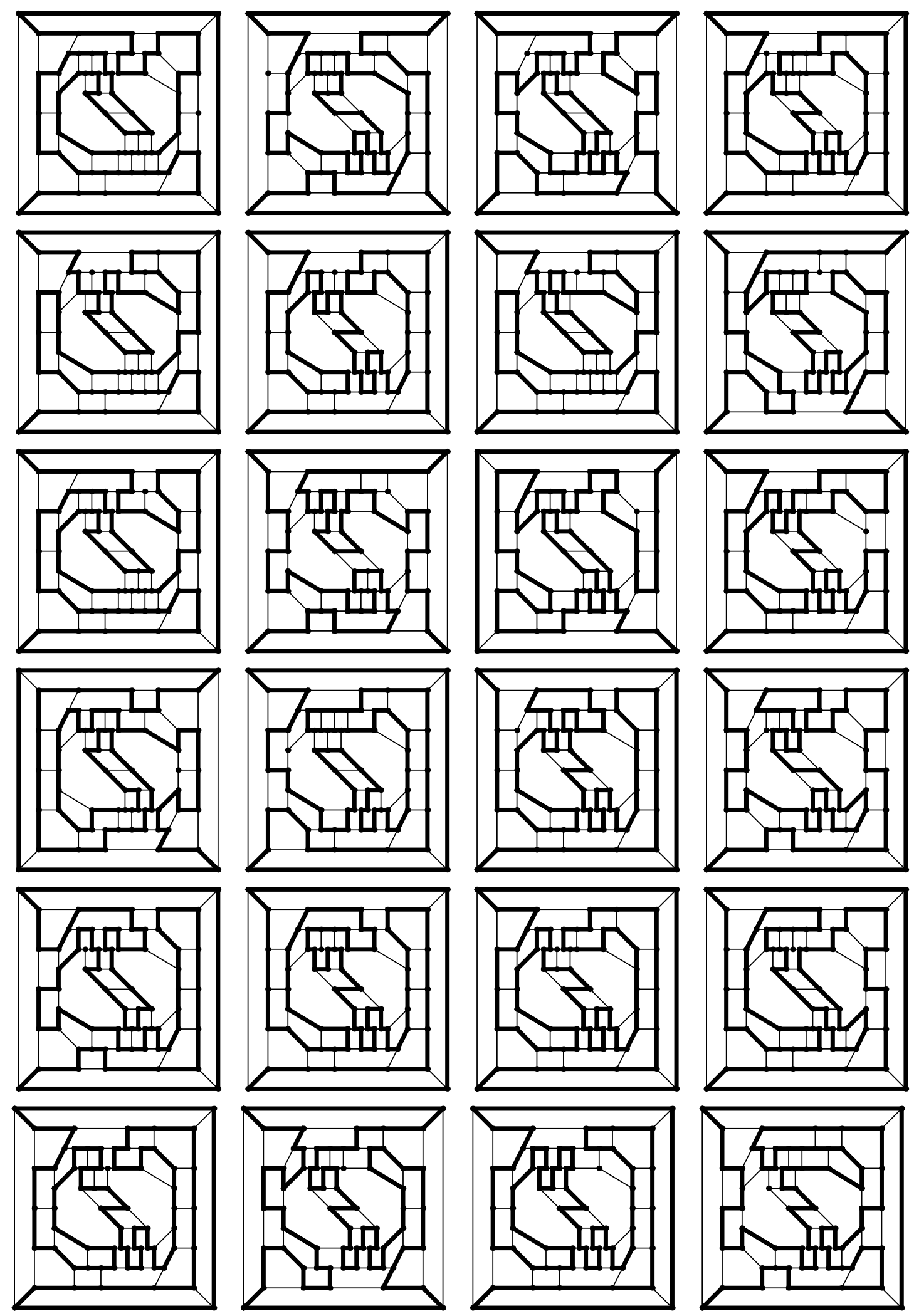

Figure 4: Hamiltonian cycles of the vertex-deleted subgraphs of $G$ (continued) 

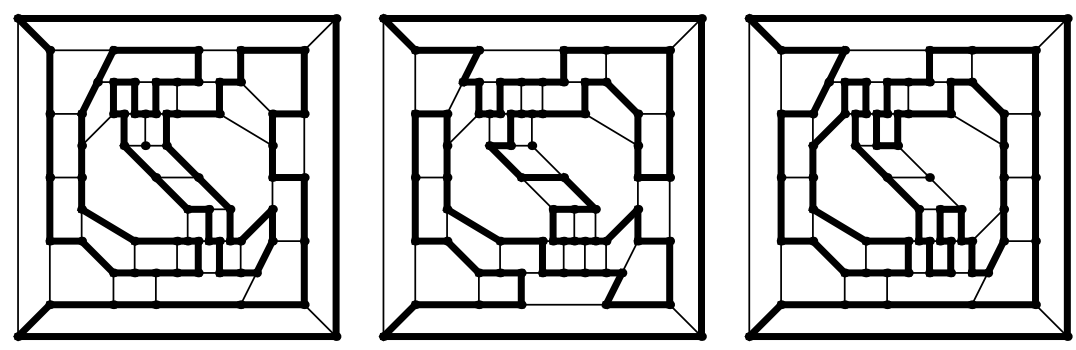

Figure 5: Hamiltonian cycles of the vertex-deleted subgraphs of $G$ (continued)
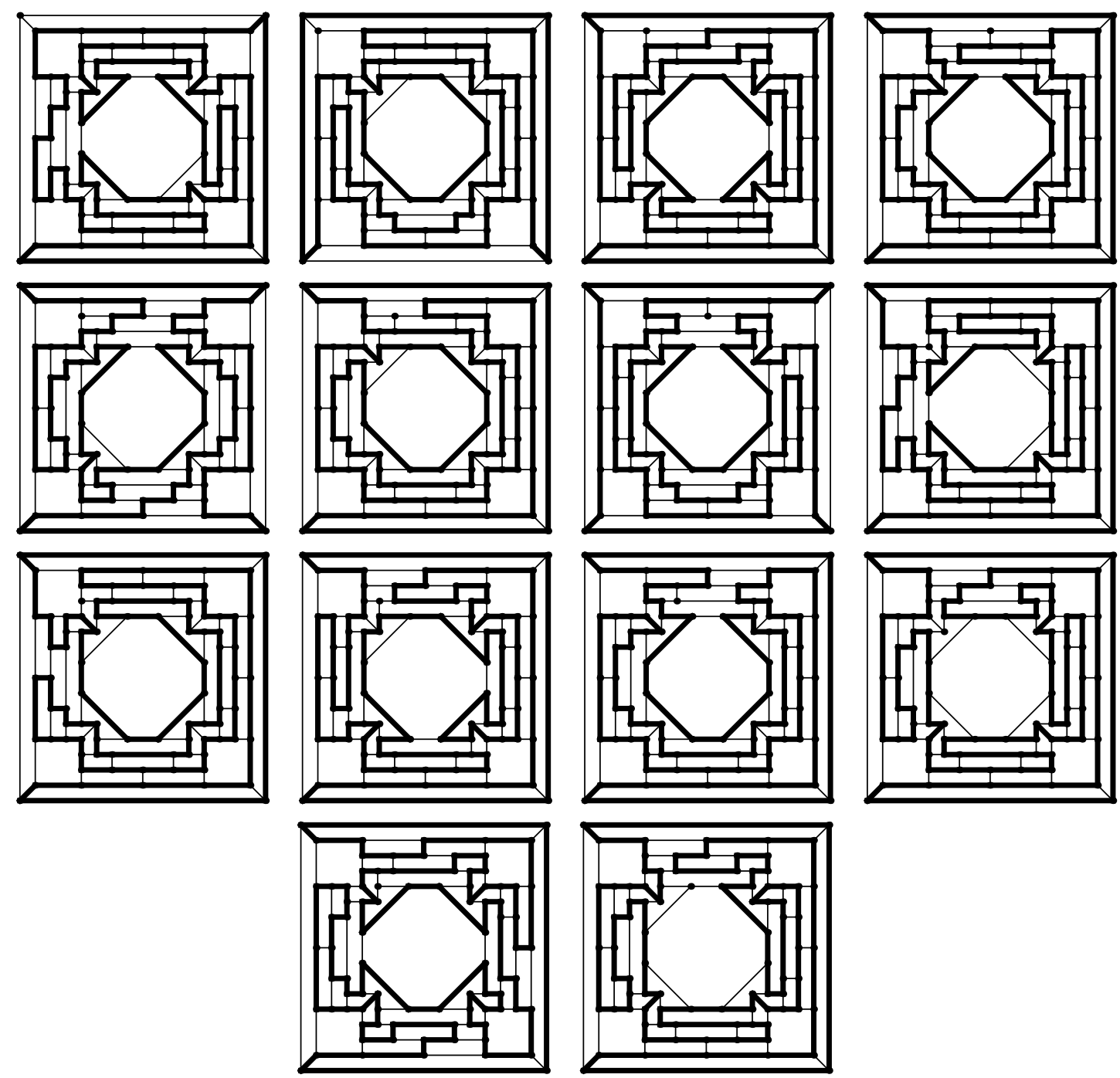

Figure 6: Hamiltonian cycles of the vertex-deleted subgraphs of $H$ 


\section{Acknowledgement}

The authors would like to thank the Suzuki Foundation for their generous support. Research of Gábor Wiener was partially supported by the Hungarian National Research Fund and by the National Office for Research and Technology (Grant Number OTKA $67651)$.

\section{References}

[1] Aldred, R. E. L., Bau, S., Holton, D. A., McKay, B. D.: Nonhamiltonian 3-connected cubic planar graphs. SIAM J. Disc. Math. 13, 25-32. (2000)

[2] Bosák, J.: Hamiltonian lines in cubic graphs. Theory of Graphs (Internat. Sympos., Rome, 1966) Gordon and Breach, New York; Dunod, Paris, 35-46. (1967)

[3] Chvátal, V.: Flip-flops in hypohamiltonian graphs. Can. Math. Bull. 16, 33-41. (1973)

[4] Grinberg, E. J.: Plane homogeneous graphs of degree three without Hamiltonian circuits. Latvian Math. Yearbook, Izdat. Zinatne, Riga 4, 51-58. (1968) (in Russian)

[5] Grünbaum, B.: Convex Polytopes. John Wiley and Sons, New York (1967)

[6] Hatzel, W.: Ein planarer hypohamiltonischer Graph mit 57 Knoten. Math. Ann. 243, 213-216. (1979)

[7] Holton, D. A., McKay, B. D.: The smallest non-hamiltonian 3-connected cubic planar graphs have 38 vertices. J. Combinatorial Theory (B) 45, 315-319. (1988)

[8] Holton, D. A., Sheehan, J.: Hypohamiltonian graphs. in: The Petersen Graph, Cambridge University Press, New York, 1993.

[9] Sousselier, R.: Problème No. 29: Le Cercle des Irascibles. Revue Française de Recherches Opérationelle 7, 405-406. (1963)

[10] Lederberg, J.: Systematics of organic molecules, graph theory and Hamiltonian circuits, Instrumentation Research Laboratory Report, no. 1040, Stanford University, Stanford, Calif., 1144 (1966)

[11] Schauerte, B., Zamfirescu, C. T.: Regular graphs in which every pair of points is missed by some longest cycle. An. Univ. Craiova, Ser. Mat. Inf. 33, 154-173. (2006)

[12] Thomassen, C.: Hypohamiltonian and hypotraceable graphs. Discrete Mathematics 9, 91-96. (1974)

[13] Thomassen, C.: On hypohamiltonian graphs. Discrete Mathematics 10, 383-390. (1974)

[14] Thomassen, C.: Planar and infinite hypohamiltonian and hypotraceable graphs. Discrete Mathematics 14, 377-389. (1976)

[15] Thomassen, C.: Hypohamiltonian graphs and digraphs. in: Theory and Applications of Graphs, Lecture Notes in Mathematics No. 642, Springer, Berlin, 557-571. (1978) 
[16] Thomassen, C.: Planar cubic hypohamiltonian and hypotraceable graphs. J. Comb. Theory B 30, 36-44. (1981)

[17] Tutte, W. T.: On hamiltonian circuits. J. London Math. Soc. 21, 98-101. (1946)

[18] Voss, H. J.: Cycles and bridges in graphs. Kluwer Academic Publishers, Dordrecht, 1990.

[19] Wiener, G., Araya, M.: On planar hypohamiltonian graphs. J. Graph Theory 67, 55-68. (2011)

[20] Zamfirescu, T.: A two-connected planar graph without concurrent longest paths. J. Comb. Theory B 13, 116-121. (1972)

[21] Zamfirescu, T.: On longest paths and circuits in graphs. Math. Scand. 38, 211-239. (1976)

[22] Zamfirescu, C., Zamfirescu, T.: A planar hypohamiltonian graph with 48 vertices. J. Graph Theory 55, 116-121. (2007) 